\title{
MIMO Capacity Gain Analysis for General Channel Models
}

\author{
Min Zhang*, Peter J. Smith ${ }^{\dagger}$, Mansoor Shafi ${ }^{\ddagger}$, Pawel A. Dmochowski ${ }^{\S}$, Jawad Mirza ${ }^{\S}$ \\ * Alcatel-Lucent, Wellington, New Zealand \\ $\dagger$ Department of Electrical and Computer Engineering, University of Canterbury, Christchurch, New Zealand \\ $\ddagger$ Telecom New Zealand, Wellington, New Zealand \\ $\S$ School of Engineering and Computer Science, Victoria University of Wellington, Wellington, New Zealand \\ email: min.hs.zhang@alcatel-lucent.com,p.smith@elec.canterbury.ac.nz, mansoor.shafi@telecom.co.nz, \\ \{pawel.dmochowski,jawad.mirza\}@ecs.vuw.ac.nz
}

\begin{abstract}
In this paper, we evaluate the capacity gains offered by MIMO systems relative to SISO by using two metrics: the ratio between the expected value of the two capacities and a weighted difference between the two capacities. We present results for these metrics for the case of a general channel, where fading could be iid, correlated Rayleigh, or any other type. We derive limiting values of the capacity metrics for low and high SNR and show via simulation their behavior at other values of SNR. These simulations provide valuable insight into the variation of the capacity metrics in the important SNR range from $-5 \mathrm{~dB}$ to $20 \mathbf{d B}$ which is commonly observed in cellular systems.
\end{abstract}

\section{INTRODUCTION}

Since the appearance of the seminal papers on MIMO by Telatar [1] and Foschini [2], numerous authors have analysed the capacity of single and multi-user MIMO systems for a wide range of fading channels, antenna configurations, receiver types, interference levels, SNR etc. (see, for example, [3][10]). In this paper, our work is motivated by a measurement study which showed that the capacity of a $2 \times 2$ MIMO link is often considerably less than twice the equivalent SISO link at typical SNR levels. Thus, we focus on an analysis of MIMO capacity gain, which is the capacity of a MIMO link relative to the equivalent SISO link. MIMO links offer a number of virtual channels equal to $m=\min \left(n_{\mathrm{T}}, n_{\mathrm{R}}\right)$, the minimum of the number of transmit and receive antennas. Hence, our capacity gain analysis is benchmarked by comparing MIMO capacity to SISO capacity scaled by $m$. This gain is considered for a range of SNR values and channel conditions with varying amounts of fading correlation at either or both ends. We consider two metrics to look at the gain, the ratio of MIMO to SISO capacity and a weighted difference between the two capacities. For both these approaches we develop limiting values at low and high SNR for arbitrary channels.

There is a wealth of papers that look at MIMO or SISO capacity although the great majority focus on Gaussian channels. A good starting point is [3], where the authors provide an overview of extensive results on the Shannon capacity of single and multiuser MIMO channels. Factors such as the availability of channel information, SNR and correlation models, are discussed in order to understand their impact on the potential capacity gains of MIMO techniques. In [9], the authors derive explicit expressions for the channel capacity using closed-form finite sums of the exponential integral for independent and identically distributed (iid) Rayleigh flat fading channels. In another paper [10], the same authors develop a closed-form expression for the mean (ergodic) capacity of the iid Rayleigh fading MIMO channel and a tight upper bound for double-sided correlated MIMO channels based on a Kronecker structure. Chiani et al. [8] derive a closed-form expression for the characteristic function $(\mathrm{CF})$ of MIMO capacity with one sided correlation, either among the transmitting antennas or among the receiving antennas, in Rayleigh-fading environments. The exact mean capacity of the MIMO system is then derived from the CF. In [7], the authors use a similar approach to [8] to derive the parameters of a Gaussian approximation to the exact capacity under one sided correlation. The impact of correlation on the change in degrees of spatial freedom of a MIMO channel is described in [6], where the mean capacity and eigenvalue distribution are also described. Loyka [5] studies MIMO systems with a large number of transmitting and receiving antennas in Rayleigh channels with an exponential correlation matrix and derives simple approximations of ergodic capacity. In [4], the author considers capacity gain and array gain, and derives an upper bound for the average capacity gain under perfect channel state information (CSI) at both ends. In [11], the variation of the capacity ratio for iid Rayleigh channels is shown for the low SNR regime and valuable insight is provided indicating that at low SNR an $n_{\mathrm{T}} \times n_{\mathrm{R}}$ system yields a power gain of $n_{\mathrm{R}}$ over a SISO system.

Whilst there are ample papers that may be used to derive the capacity ratio under certain channel conditions, there is no single work that analyses the ratio for general channel conditions. Furthermore, there is a scarcity of published information on capacity difference. Closed form expressions for both the ratio and difference that are valid for all SNR values are intractable for general channels. Therefore, we resort to limiting values at low and high SNR. At low SNR there is a power gain regime as noted by [11], but at high SNR there is a spatial degrees of freedom gain regime. The change from one regime to another as the SNR increases is interesting to observe in our simulations. Motivated by these issues, we make the following contributions in this paper: 
- We evaluate limiting values (for low and high SNR) for the capacity ratio and difference for arbitrary channels for both equal power and water filling (the later assuming perfect CSI at the transmitter). We derive an exact value of capacity difference at infinite SNR, $D_{\infty}$.

- We analyze the effects of system size and correlation on these limits as well as simulating the metrics for moderate SNR levels.

- We demonstrate that the MIMO ratio gain can drop well below $m$ for typical SNR values and is further reduced by correlation.

\section{System Model And Performance Metrics}

Consider a single-user MIMO link with $n_{\mathrm{T}}$ transmit antennas, $n_{\mathrm{R}}$ receive antennas, a flat fading channel matrix, $\mathbf{H}$, total transmit power, $P$, and a link SNR of $\rho=P / \sigma^{2}$, where $\sigma^{2}$ is the power of the additive white Gaussian noise at each receive antenna. For this system, the standard capacity equations are

$$
\begin{gathered}
C_{\mathrm{M}}=\log _{2}\left(\left|\mathbf{I}+\frac{\rho}{n_{\mathrm{T}}} \mathbf{W}\right|\right), \\
\tilde{C}_{\mathrm{M}}=\sum_{i=1}^{\operatorname{rank} \mathbf{H}} \log _{2}\left(1+\frac{1}{\sigma^{2}}\left(\lambda_{i} \mu-\sigma^{2}\right)^{+}\right)
\end{gathered}
$$

where $C_{\mathrm{M}}$ is the MIMO capacity with no CSI at the transmitter, $\tilde{C}_{\mathrm{M}}$ is the waterfilling capacity with perfect CSI at the transmitter, $(a)^{+}=\max (a, 0), \mu$ is the water level, and $\mathbf{W}$ represents the $m \times m$ matrix

$$
\mathbf{W}=\left\{\begin{array}{ll}
\mathbf{H H}^{\dagger} & n_{\mathrm{R}} \leq n_{\mathrm{T}} \\
\mathbf{H}^{\dagger} \mathbf{H} & n_{\mathrm{T}} \leq n_{\mathrm{R}}
\end{array},\right.
$$

with $\lambda_{1} \geq \lambda_{2} \geq \ldots \geq \lambda_{m}$ denoting its $m$ eigenvalues.

The focus of this paper is on the gains offered by MIMO over the corresponding SISO link. To evaluate these gains we consider the difference measure

$$
D=\mathbb{E}\left(C_{\mathrm{M}}\right)-m \mathbb{E}\left(C_{\mathrm{S}}\right),
$$

where $C_{\mathrm{S}}$ is the SISO capacity,

$$
C_{\mathrm{S}}=\log _{2}\left(1+\rho|h|^{2}\right),
$$

and $h$ is the SISO channel coefficient drawn from the same channel model as the MIMO system. We also consider the relative gains via the ratio

$$
R=\frac{\mathbb{E}\left(C_{\mathrm{M}}\right)}{\mathbb{E}\left(C_{\mathrm{S}}\right)} .
$$

Equivalent measures, $\tilde{D}$ and $\tilde{R}$, are defined for the case where perfect CSI is available at the transmitter.

\section{A. Channel Models}

In this paper, we consider a range of channel models for the single-user MIMO link. These models include a general channel model, iid and correlated Rayleigh fading and a fully correlated channel model. For the general model, we have the channel matrix $\mathbf{H}$, where the only assumptions are that the matrix is normalized, $\mathbb{E}\left[\left|\mathbf{H}_{i j}\right|^{2}\right]=1$, and the entries of $\mathbf{H}$ do not have perfect correlation. Hence, $\operatorname{corr}\left(\mathbf{H}_{i, j}, \mathbf{H}_{k, l}\right) \neq 1$ for $(i, j) \neq(k, l)$. The general channel model makes no distributional assumptions and therefore incorporates spatial, geometric models as well as statistical models such as Rayleigh, Ricean, Nakagami, etc.

The Rayleigh model is defined by

$$
\mathbf{H}=\mathbf{R}_{\mathrm{R}}^{1 / 2} \mathbf{U} \mathbf{R}_{\mathrm{T}}^{1 / 2},
$$

where the entries of $\mathbf{U}$ are iid $\mathcal{C N}(0,1)$ and $\mathbf{R}_{R}, \mathbf{R}_{\mathrm{T}}$ are the correlation matrices at the receiver and transmitter respectively. Hence, the standard Kronecker model is assumed [6]. When $\mathbf{R}_{\mathrm{R}}=\mathbf{R}_{\mathrm{T}}=\mathbf{I}$ we have iid Rayleigh fading. The fully correlated channel is defined by

$$
\mathbf{H}=h_{1} \mathbf{1}_{n_{\mathrm{R}} \times n_{\mathrm{T}}},
$$

where $h_{1}$ is a single channel coefficient that is identical for all $n_{\mathrm{T}} n_{\mathrm{R}}$ links, giving perfect correlation between the elements of $\mathbf{H}$. Although a rather artificial model, it is a useful tool to gain insight into the effects of correlation. Note that all channel models have $\mathbb{E}\left[\left|\mathbf{H}_{i, j}\right|^{2}\right]=1$ so that $\mathbb{E}\left[\operatorname{tr}\left(\mathbf{H H}^{\dagger}\right)\right]=n_{\mathrm{R}} n_{\mathrm{T}}$.

\section{B. Mathematical Preliminaries}

The following straightforward results are necessary in the analysis of Section III. For the general channel model at high SNR $(\rho \rightarrow \infty)$ we have

$$
\frac{\left|\mathbf{I}+\frac{\rho}{n_{\mathrm{T}}} \mathbf{W}\right|}{\left(\frac{\rho}{n_{\mathrm{T}}}\right)^{m}|\mathbf{W}|} \approx 1 .
$$

Note that $|\mathbf{W}|>0$ with probability 1 for the general channel model. For the SISO channel at high SNR

$$
\frac{\left(1+\rho|h|^{2}\right)^{m}}{\left(\rho|h|^{2}\right)^{m}} \approx 1 .
$$

At low SNR $(\rho \rightarrow 0)$ we have

$$
\left|\mathbf{I}+\frac{\rho}{n_{\mathrm{T}}} \mathbf{W}\right| \approx 1+\frac{\rho}{n_{\mathrm{T}}} \operatorname{tr}(\mathbf{W}),
$$

and the logarithmic property

$$
\log _{2}(1+\epsilon) \approx \epsilon \log _{2} e
$$

for small $\epsilon$, is also useful. Finally, for the fully correlated model, a little algebra shows that

$$
\left|\mathbf{I}+\frac{\rho}{n_{\mathrm{T}}} \mathbf{W}\right|=1+\rho n_{\mathrm{R}}\left|h_{1}\right|^{2} .
$$

\section{ANALYSIS}

\section{A. The General Channel Model}

1) No CSI at the transmitter: The difference measure, $D$, in (4) can be evaluated at low and high SNR as shown below. Using (11) and (12) in (4) gives, in the low SNR case,

$$
D \approx \mathbb{E}\left[\log _{2}\left(1+\frac{\rho}{n_{\mathrm{T}}} \operatorname{tr}(\mathbf{W})\right)\right]-m \log _{2} e \mathbb{E}\left[\rho|h|^{2}\right],
$$


as $\rho \rightarrow 0$. Using (12), $\mathbb{E}[\operatorname{tr}(\mathbf{W})]=n_{\mathrm{R}} n_{\mathrm{T}}$ and $\mathbb{E}\left[|h|^{2}\right]=1$ gives

$$
D \approx \rho\left(n_{\mathrm{R}}-m\right) \log _{2} e .
$$

Hence, $D \rightarrow 0$ as $\rho \rightarrow 0$ and $D>0$ for small $\rho$ if $n_{\mathrm{R}}>n_{\mathrm{T}}$.

When $n_{\mathrm{R}} \leq n_{\mathrm{T}}$, (15) does not indicate the sign of $D$ for small $\rho$, since $n_{\mathrm{R}}-m=0$. Taking higher order terms in $\rho$ in (14) shows that $D>0$ for small $\rho$ if $n_{\mathrm{T}}>n_{\mathrm{R}}$ and $D<0$ if $n_{\mathrm{T}}=n_{\mathrm{R}}$. Proof is omitted for reasons of space.

At high SNR, it is more convenient to express $D$ as

$$
D=\mathbb{E}\left[\log _{2}\left(\left(1+\rho|h|^{2}\right)^{-m}\left|\mathbf{I}+\frac{\rho}{n_{\mathrm{T}}} \mathbf{W}\right|\right)\right] .
$$

Using (9) and (10) in (16) gives, at high SNR,

$$
\begin{aligned}
D & \approx \mathbb{E}\left[\log _{2}\left(\left(\rho|h|^{2}\right)^{-m}\left(\frac{\rho}{n_{\mathrm{T}}}\right)^{m}|\mathbf{W}|\right)\right] \\
& =\mathbb{E}\left[\log _{2}\left(\left(n_{\mathrm{T}}|h|^{2}\right)^{-m}|\mathbf{W}|\right)\right] \triangleq D_{\infty} .
\end{aligned}
$$

Hence $D \rightarrow D_{\infty}$ as $\rho \rightarrow \infty$, where $D_{\infty}$ is a constant which can be positive or negative depending on the channel statistics. It is well known that correlation tends to reduce $|\mathbf{W}|$. Hence, from (17), we observe that correlation will also tend to reduce $D_{\infty}$ and will thus have a corresponding effect in reducing $D$.

The limiting results for $D$ have a direct application to the limits of $R$, since $R$ can be expressed as

$$
R=\frac{D}{\mathbb{E}\left[C_{\mathrm{S}}\right]}+m \text {. }
$$

Hence, at low SNR, $D \approx \rho\left(n_{\mathrm{R}}-m\right) \log _{2} e$ and $C_{\mathrm{S}} \approx$ $\rho|h|^{2} \log _{2} e$, so that $\mathbb{E}\left[C_{\mathrm{S}}\right] \approx \rho \log _{2} e$ giving

$$
R \rightarrow n_{\mathrm{R}}
$$

as $\rho \rightarrow 0$. At high SNR, $D \rightarrow D_{\infty}$ and $\mathbb{E}\left[C_{\mathrm{S}}\right]$ grows monotonically so that $R \rightarrow m$ as $\rho \rightarrow \infty$.

2) Perfect CSI at the transmitter: It is well known that at low SNR, $\rho \rightarrow 0$, waterfilling only uses the dominant eigenchannel and at high SNR, $\rho \rightarrow \infty$, waterfilling allocates power evenly amongst the eigenchannels. These properties enable the limiting values of $\tilde{D}$ and $\tilde{R}$ to be identified.

From (2), as $\rho \rightarrow 0$ and only the dominant eigenchannel is used, we have $\mu-\frac{1}{\lambda_{1}}=\rho$ and

$$
\tilde{C}_{\mathrm{M}}=\log _{2}\left(1+\rho \lambda_{1}\right) \approx \rho \lambda_{1} \log _{2} e .
$$

Substituting (20) in $\tilde{D}$ and $\tilde{R}$ gives

$$
\tilde{D} \approx \rho\left(\mathbb{E}\left(\lambda_{1}\right)-m\right) \log _{2} e
$$

and

$$
\tilde{R} \approx \mathbb{E}\left(\lambda_{1}\right) .
$$

Solutions for $\mathbb{E}\left(\lambda_{1}\right)$ are known for iid and semi-correlated Rayleigh channels [12]. Hence, at low SNR, $\tilde{R} \rightarrow \mathbb{E}\left(\lambda_{1}\right)$ and $\tilde{D} \rightarrow 0$ as $\rho \rightarrow 0$.

At high SNR, it is known that the $m$ eigenchannels are allocated equal power. Hence, the capacity becomes

$$
\begin{aligned}
\tilde{C}_{\mathrm{M}} & \approx \sum_{i=1}^{m} \log _{2}\left(1+\frac{\rho}{m} \lambda_{i}\right) \\
& =\sum_{i=1}^{m} \log _{2}\left(1+\left(\frac{\rho n_{\mathrm{T}}}{m}\right) \frac{\lambda_{i}}{n_{\mathrm{T}}}\right) .
\end{aligned}
$$

Now (23) is the capacity of an $n_{\mathrm{R}} \times n_{\mathrm{T}}$ channel with no CSI at the transmitter and SNR $=\rho n_{\mathrm{T}} / m$. Using (17) and (18) with this new SNR value gives

$$
\tilde{D} \rightarrow \mathbb{E}\left[\log _{2}\left(\left(m|h|^{2}\right)^{-m}|\mathbf{W}|\right)\right]
$$

and $\tilde{R} \rightarrow m$ as $\rho \rightarrow \infty$. The capacity boost from waterfilling to the high SNR difference measure can be evaluated as $\Delta=$ $\lim _{\rho \rightarrow \infty}(\tilde{D}-D)$. Substituting (24) and (17) into $\Delta$ gives

$$
\Delta=m \log _{2}\left(\frac{n_{\mathrm{T}}}{m}\right)
$$

and thus the capacity difference at high SNR becomes

$$
\tilde{D} \rightarrow D_{\infty}+m \log _{2}\left(\frac{n_{\mathrm{T}}}{m}\right) .
$$

\section{B. The Fully Correlated Channel Model}

1) No CSI at the transmitter: For the fully correlated case, using (13) in (4) gives

$$
D=\mathbb{E}\left[\log _{2}\left(1+\rho n_{\mathrm{R}}\left|h_{1}\right|^{2}\right)\right]-m \mathbb{E}\left[\log _{2}\left(1+\rho|h|^{2}\right)\right] .
$$

Using (12) in (27) gives, at low SNR,

$$
\begin{aligned}
D & \approx\left(\mathbb{E}\left[\rho n_{\mathrm{R}}\left|h_{1}\right|^{2}\right]-m \mathbb{E}\left[\rho n_{\mathrm{R}}|h|^{2}\right]\right) \log _{2} e \\
& =\rho\left(n_{\mathrm{R}}-m\right) \log _{2} e .
\end{aligned}
$$

At high SNR, (27) becomes

$$
\begin{aligned}
D & \approx \mathbb{E}\left[\log _{2}\left(\rho n_{\mathrm{R}}\left|h_{1}\right|^{2}\right)\right]-m \mathbb{E}\left[\log _{2}\left(\rho|h|^{2}\right)\right] \\
& =-(m-1) \log _{2} \rho+c,
\end{aligned}
$$

for some constant $c$. Hence, the low SNR behaviour is the same as for the general channel. However, at high SNR, $D$ becomes increasingly negative, heading to $-\infty$ as $\rho \rightarrow \infty$ at a gradient of $(m-1) \frac{\log _{2} 10}{10} \mathrm{bps} / \mathrm{Hz} / \mathrm{dB}$. In contrast, $D \rightarrow D_{\infty}$ for the general channel.

For $R$, we use (13) in (6) to give

$$
R=\frac{\mathbb{E}\left[\log _{2}\left(1+\rho n_{\mathrm{R}}\left|h_{1}\right|^{2}\right)\right]}{\mathbb{E}\left[\log _{2}\left(1+\rho|h|^{2}\right)\right]} .
$$

At low SNR, using (12) in (30) gives $R \rightarrow n_{\mathrm{R}}$ as $\rho \rightarrow 0$. At high SNR, the $\log _{2} \rho$ term dominates in both the numerator and denominator of (30) so that $R \rightarrow 1$ as $\rho \rightarrow \infty$.

2) Perfect CSI at the transmitter: For the fully correlated channel, $\mathbf{W}$ has a single non-zero eigenvalue, with $\mathbb{E}\left(\lambda_{1}\right)=$ $n_{\mathrm{T}} n_{\mathrm{R}}$. Substituting $\mathbb{E}\left(\lambda_{1}\right)$ in (21) and (22) gives

$$
\tilde{D} \approx \rho\left(n_{\mathrm{T}} n_{\mathrm{R}}-m\right) \log _{2} e
$$

and

$$
\tilde{R} \approx n_{\mathrm{T}} n_{\mathrm{R}},
$$


TABLE I

SUMMARY OF CAPACITY GAIN RESULTS: RATIO, $R$, AND DIFFERENCE, $D$, FOR LIMITING $\rho$

\begin{tabular}{|c|c|c|c|c|c|}
\hline \multicolumn{2}{|c|}{} & \multicolumn{2}{c|}{ General Channel } & \multicolumn{2}{c|}{ Fully Correlated Channel } \\
\cline { 3 - 6 } \multicolumn{2}{c|}{} & $\rho \rightarrow 0$ & $\rho \rightarrow \infty$ & $\rho \rightarrow 0$ & $\rho \rightarrow \infty$ \\
\hline \multirow{2}{*}{ No CSI } & $R$ & $n_{\mathrm{R}}$ & $m$ & $n_{\mathrm{R}}$ & 1 \\
\cline { 2 - 6 } & $D$ & 0 & $D_{\infty}$ & 0 & $-\infty$ \\
\hline \multirow{2}{*}{ Full CSI } & $\tilde{R}$ & $\mathbb{E}\left(\lambda_{1}\right)$ & $m$ & $n_{\mathrm{T}} n_{\mathrm{R}}$ & 1 \\
\cline { 2 - 6 } & $\tilde{D}$ & 0 & $D_{\infty}+m \log _{2} \frac{n_{\mathrm{T}}}{m}$ & 0 & $\log _{2} n_{\mathrm{T}} n_{\mathrm{R}}(m=1),-\infty(m>1)$ \\
\hline
\end{tabular}

TABLE II

HIGH SNR CAPACITY LIMITS FOR IID MIMO,SIMO AND MISO ANTENNA CONFIGURATIONS

\begin{tabular}{|l|l|}
\hline Antenna Configurations & $D_{\infty}$ \\
\hline$n_{\mathrm{T}}=1, n_{\mathrm{R}}>1$ & $\log _{2}(e) \sum_{j=1}^{n_{\mathrm{R}}-1} \frac{1}{j}$ \\
\hline$n_{\mathrm{T}}>1, n_{\mathrm{R}}=1$ & $\log _{2}(e) \sum_{j=1}^{n_{\mathrm{T}}-1} \frac{1}{j}-\log _{2} n_{\mathrm{T}}$ \\
\hline$n_{\mathrm{T}}=n_{\mathrm{R}}=2$ & $\log _{2}(e)-2=-0.56$ \\
\hline$n_{\mathrm{T}}=4, n_{\mathrm{R}}=2$ & $10 \log _{2}(e) / 3-4=0.81$ \\
\hline$n_{\mathrm{T}}=2, n_{\mathrm{R}}=4$ & $10 \log _{2}(e) / 3-2=2.81$ \\
\hline$n_{\mathrm{T}}=n_{\mathrm{R}}=4$ & $13 \log _{2}(e) / 3-8=-1.75$ \\
\hline
\end{tabular}

at low SNR. Hence $\tilde{D} \rightarrow 0$ and $\tilde{R} \rightarrow n_{\mathrm{T}} n_{\mathrm{R}}$ as $\rho \rightarrow 0$.

At high SNR, $\tilde{C}_{\mathrm{M}}=\log _{2}\left(1+\rho \lambda_{1}\right)$, so that

$$
\begin{aligned}
\tilde{D} & \approx \mathbb{E}\left[\log _{2}\left(\rho \lambda_{1}\right)\right]-m \mathbb{E}\left[\log _{2}\left(\rho|h|^{2}\right)\right] \\
& =\log _{2} n_{\mathrm{T}} n_{\mathrm{R}}+(1-m) \log _{2} \rho .
\end{aligned}
$$

Hence, as $\rho \rightarrow \infty, \tilde{D} \rightarrow \log _{2} n_{\mathrm{T}} n_{\mathrm{R}}$ if $m=1$ and $\tilde{D} \rightarrow-\infty$ if $m>1$.

Noting that for high SNR, $\mathbb{E}\left[\tilde{C}_{\mathrm{M}}\right] \approx \log _{2} \rho$, using (33) and (18) we obtain $\tilde{R} \rightarrow 1$ as $\rho \rightarrow \infty$. The results of Sections III-A and III-B are summarized in Table I.

\section{Evaluation of $D_{\infty}$ for Rayleigh Channels}

It is possible to derive a closed form expression for $D_{\infty}$ in the Rayleigh case. This enables the evaluation of the high SNR capacity difference for equal power and water filling systems ${ }^{1}$.

1) $D_{\infty}$ for iid Channel: In the case of iid Rayleigh channels, using the closed-form expression for the ergodic MIMO capacity [9] and the SISO capacity, it can be shown that the high SNR asymptotic capacity difference, $D_{\infty}$, is given by

$$
\begin{aligned}
D_{\infty}= & \log _{2}(e) \sum_{k=0}^{m-1} \sum_{l=0}^{k} \sum_{i=0}^{2 l}\left\{\frac{(-1)^{i}(2 l) !\left(\left|n_{\mathrm{T}}-n_{\mathrm{R}}\right|+i\right) !}{2^{2 k-i} l ! i !\left(\left|n_{\mathrm{T}}-n_{\mathrm{R}}\right|+l\right) !}\right. \\
& \left.\times\left(\begin{array}{c}
2 k-2 l \\
k-l
\end{array}\right)\left(\begin{array}{c}
2 l+2\left|n_{\mathrm{T}}-n_{\mathrm{R}}\right| \\
2 l-i
\end{array}\right) \sum_{j=1}^{\left|n_{\mathrm{T}}-n_{\mathrm{R}}\right|+i}\left(\frac{1}{j}\right)\right\} \\
& -m \log _{2} n_{\mathrm{T}} .
\end{aligned}
$$

Table II shows values for $D_{\infty}$ calculated from (34) for a number of specific antenna configurations. More compact expressions for a number of specific antenna configurations can be obtained by means of the approximation methods in

\footnotetext{
${ }^{1}$ Due to space constraints, details of the derivation of $D_{\infty}$ are deferred to the journal version of this paper.
}

[10]. For $n_{\mathrm{T}}=n_{\mathrm{R}}=N, D_{\infty}$ for iid MIMO Rayleigh channels can be approximated and simplified as

$$
D_{\infty} \approx(N-1)(\gamma-1) \log _{2} e,
$$

where $\gamma$ is the Euler constant. Equation (35) suggests a linear increase in $D_{\infty}$ with respect to the number of transmit and receive antennas.

For $n_{\mathrm{T}}>n_{\mathrm{R}}$ and using $z=n_{\mathrm{T}} / n_{\mathrm{R}}, D_{\infty}$ can be approximated by

$$
\begin{aligned}
D_{\infty} & \approx \log _{2} e \sum_{j=1}^{\lceil z\rceil-1} \frac{1}{j}-\log _{2}\lceil z\rceil \\
& +\left(n_{\mathrm{R}}-1\right)\left\{(z-1) \log _{2} \frac{z}{z-1}+(\gamma-1) \log _{2} e\right\}
\end{aligned}
$$

where $\lceil\cdot\rceil$ is the ceiling function. Consider a MIMO system with $z=2$ (such as $n_{\mathrm{T}}=4, n_{\mathrm{R}}=2$ ). Here, $D_{\infty}$ can be simplified as $D_{\infty} \approx 0.44+0.39\left(n_{\mathrm{R}}-1\right)$.

For $n_{\mathrm{T}}<n_{\mathrm{R}}$ and using $z=n_{\mathrm{R}} / n_{\mathrm{T}}$, the capacity difference can be approximated as

$$
\begin{aligned}
D_{\infty} & \approx \log _{2} e \sum_{j=1}^{\lceil z\rceil-1} \frac{1}{j}+\left(n_{\mathrm{T}}-1\right) \\
& \times\left\{z \log _{2} z+(1-z) \log _{2}(z-1)+(\gamma-1) \log _{2} e\right\} .
\end{aligned}
$$

Consider again the case where $z=2$ (such as $n_{\mathrm{T}}=2, n_{\mathrm{R}}=$ 4). Here (37) becomes $D_{\infty} \approx 1.44+1.39\left(n_{\mathrm{T}}-1\right)$.

The approximations in (35) to (37) have increased accuracy when $z$ is an integer or is much larger than 1 .

2) $D_{\infty}$ for Correlated Channels: Assuming the Kronecker correlation model in (7), a simple approximation for $D_{\infty}$ exists in the case where $n_{\mathrm{T}}=n_{\mathrm{R}}=N$. It is well known that for the MIMO channel in (7), the high SNR approximation for the ergodic capacity is given by

$$
\begin{aligned}
C_{\mathrm{M}} & \approx \mathbb{E}\left[\log _{2}\left|\frac{\rho}{n_{\mathrm{T}}} \mathbf{R}_{\mathrm{R}}^{1 / 2} \mathbf{H R}_{\mathrm{T}}^{1 / 2} \mathbf{R}_{\mathrm{T}}^{1 / 2}{ }^{\dagger} \mathbf{H}^{\dagger} \mathbf{R}_{\mathrm{R}}^{1 / 2^{\dagger}}\right|\right] \\
& =\log _{2}\left|\mathbf{R}_{\mathrm{R}}\right|+\log _{2}\left|\mathbf{R}_{\mathrm{T}}\right|+\mathbb{E}\left[\log _{2}\left|\frac{\rho}{n_{\mathrm{T}}} \mathbf{W}\right|\right] .
\end{aligned}
$$

The last term in (38) represents the ergodic capacity of an iid MIMO Rayleigh channel at high SNR. Thus, the limiting capacity difference for an $\mathrm{N} \times \mathrm{N}$ correlated MIMO channel can be simply expressed as

$$
D_{\infty}^{\text {corr }}=D_{\infty}+\log _{2}\left|\mathbf{R}_{\mathrm{R}}\right|+\log _{2}\left|\mathbf{R}_{\mathrm{T}}\right|,
$$


where $D_{\infty}$ is given by (34) or approximated by (35). Corresponding results for $n_{\mathrm{R}} \neq n_{\mathrm{T}}$ are possible, but are omitted for reasons of space.

\section{Simulation Results}

In this section, we present simulation results for MIMO capacity gain and difference for the scenarios described in Section III. Specifically, we consider equal power (no CSI) and water-filling (perfect CSI) power allocation for Rayleigh channels which are iid, correlated at both Tx- and Rx-side or fully correlated. In the case of $\mathrm{Tx}$ - and Rx-side correlation, the elements of $\mathbf{R}_{\mathrm{R}}$ and $\mathbf{R}_{\mathrm{T}}$ are given by $\left(\mathbf{R}_{\mathrm{R}}\right)_{i, j}=\left(\mathbf{R}_{\mathrm{T}}\right)_{i, j}=$ $r^{|i-j|}$, the so-called exponential correlation structure [5]. The antenna configuration and the correlation value, $r$, are indicated in the relevant figures.

Figures 1 and 2 show the capacity gain, $R$, and difference, $D$, for equal power distribution across all eigenchannels for two-sided correlation. The results are consistent with the analytical derivations given in Section III-A1, specifically $R \rightarrow n_{\mathrm{R}}$ as $\rho \rightarrow 0, R \rightarrow m$ as $\rho \rightarrow \infty, D \rightarrow 0$ as $\rho \rightarrow 0$ and $D \rightarrow D_{\infty}$ as $\rho \rightarrow \infty$. The asymptotic values of $D_{\infty}$ in Fig. 2 are consistent with those given in Table II. The interesting troughs in $R$ for $2 \times 2$ and $4 \times 4$ systems follow since the limits for both low and high SNR are $m$, whereas $D<0$ for small $\rho$, as discussed below (15). This indicates an initial drop in the value of $R$ before returning to the limit. Similarly, for a $4 \times 2$ system we see a peak in $R$ as the limits are $m$ and $D>0$ for small $\rho$. These trends are important since the peaks and troughs occur in the -5 to $20 \mathrm{~dB}$ range, the practical range of operation. Hence, for symmetric systems, the desired capacity scaling of $m$ relative to SISO is not achieved and correlation will further reduce the gain.

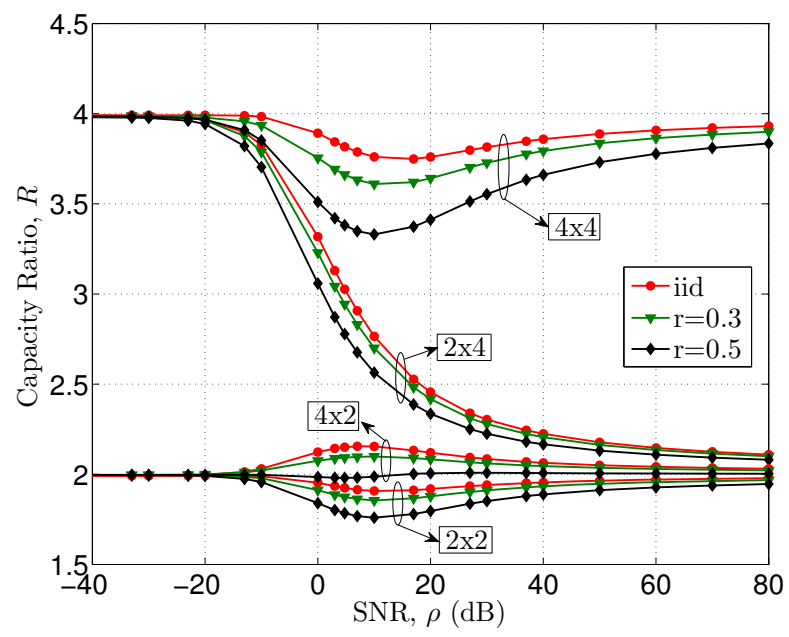

Fig. 1. $R$ with equal power distribution and $\mathrm{Tx}, \mathrm{Rx}$-side correlation.

Figures 3 and 4 show the capacity gain and difference, respectively, for water filling power allocation in a two-sided correlation scenario. The asymptotic results agree with the theory in Section III-A2. At high SNR, $\tilde{R} \rightarrow m$ as $\rho \rightarrow \infty$. There are two kinds of gain; the power gain regime at low SNR

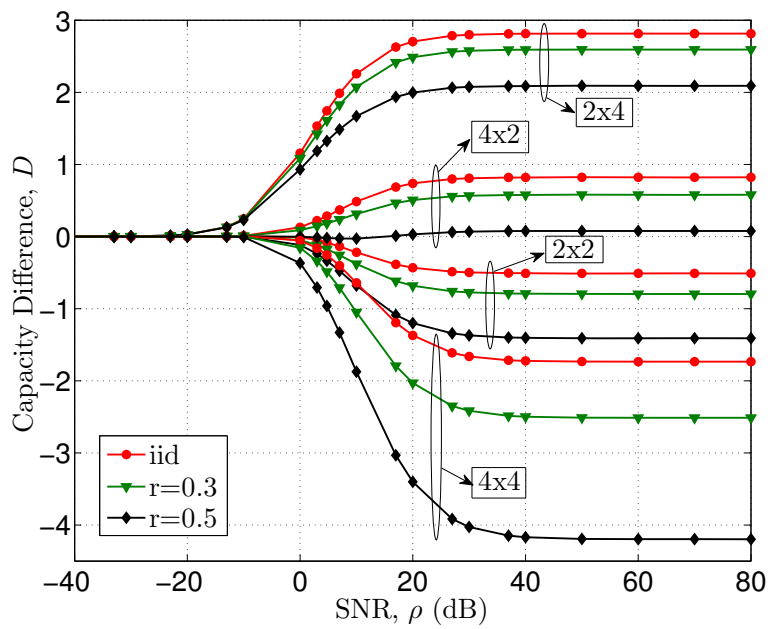

Fig. 2. $D$ with equal power distribution and $\mathrm{Tx}, \mathrm{Rx}$-side correlation.

and the spatial degrees of freedom gain at high SNR. Figures 3 and 4 show the limiting values of these two regimes. However, SNR values in a cellular network are typically around $-5 \mathrm{~dB}$ to $20 \mathrm{~dB}$. In this range of SNR, a change of regime from the power gain to degree of freedom gain is evident. Also, depending upon $n_{\mathrm{R}}$ and $n_{\mathrm{T}}$, the capacity ratio could be more or less than the limiting values as a function of SNR. For $2 \times 2$ and $4 \times 4$ systems the pattern follows from (21) and (26). As $\rho \rightarrow 0, \tilde{D} \rightarrow 0$ while $\tilde{D}>0$ for small $\rho$ as shown in (21). As $\rho \rightarrow \infty, \tilde{D} \rightarrow D_{\infty}$ from (26) and $D_{\infty}$ is negative (see Table II). Hence, we observe the initial rise followed by a drop to a negative limit. Focusing on the difference measure at high SNR, the improvement due to water filling, as given by (25), is $\Delta=2$ for $n_{\mathrm{T}}=4, n_{\mathrm{R}}=2$ and zero for the other antenna configurations shown. Hence, relative to the equal power results in Fig. 2, $\tilde{D} \rightarrow D_{\infty}+2$ for $n_{\mathrm{T}}=4$, $n_{\mathrm{R}}=2$ and $\tilde{D} \rightarrow D_{\infty}$ for the other cases.

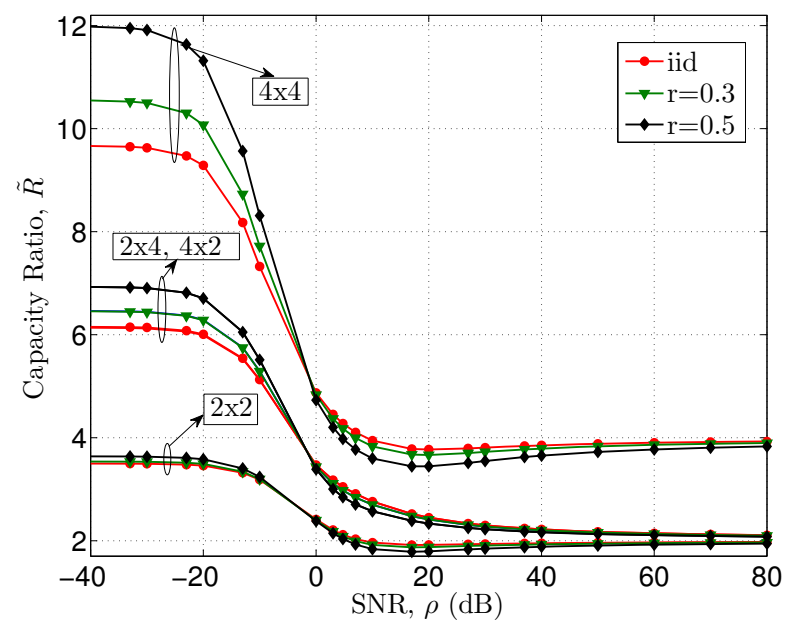

Fig. 3. $\tilde{R}$ with water filling and $\mathrm{Tx}, \mathrm{Rx}$-side correlation.

Figures 5 and 6 show the capacity ratio and difference, 


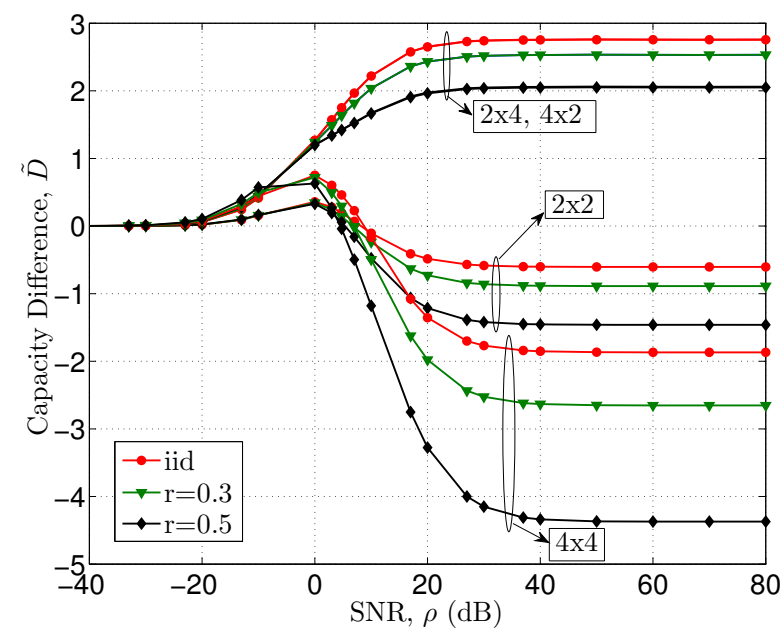

Fig. 4. $\tilde{D}$ with water filling and $\mathrm{Tx}, \mathrm{Rx}$-side correlation.

respectively, for the fully correlated channel case with equal power distribution and water-filling. In the case of equal power distribution, as noted in Section III-B1, the low SNR behaviour is identical to the general channel case. At high SNR, we observe that $R \rightarrow 1$ as $\rho \rightarrow \infty$ and $D \rightarrow-\infty$ as $\rho \rightarrow \infty$ with a gradient of $\frac{(m-1) \log _{2} 10}{10}$. These results are consistent with those derived in Section III-B1. For the case of perfect CSI at the Tx, as derived in Section III-B2, $\tilde{R} \rightarrow n_{\mathrm{T}} n_{\mathrm{R}}$ as $\rho \rightarrow 0$ and $\tilde{R} \rightarrow 1$ as $\rho \rightarrow \infty$. The capacity difference $\tilde{D} \rightarrow 0$ as $\rho \rightarrow 0$ and $\tilde{D} \rightarrow-\infty$ as $\rho \rightarrow \infty$ for the cases of $m=2,4$ as shown.

The fully correlated results have $R$ and $\tilde{R}$ decaying monotonically and $D$ and $\tilde{D}$ diverging to $-\infty$. This further explains the trends in Figs 1 and 2 where increasing levels of correlation cause a drop in the $R, D$ curves.

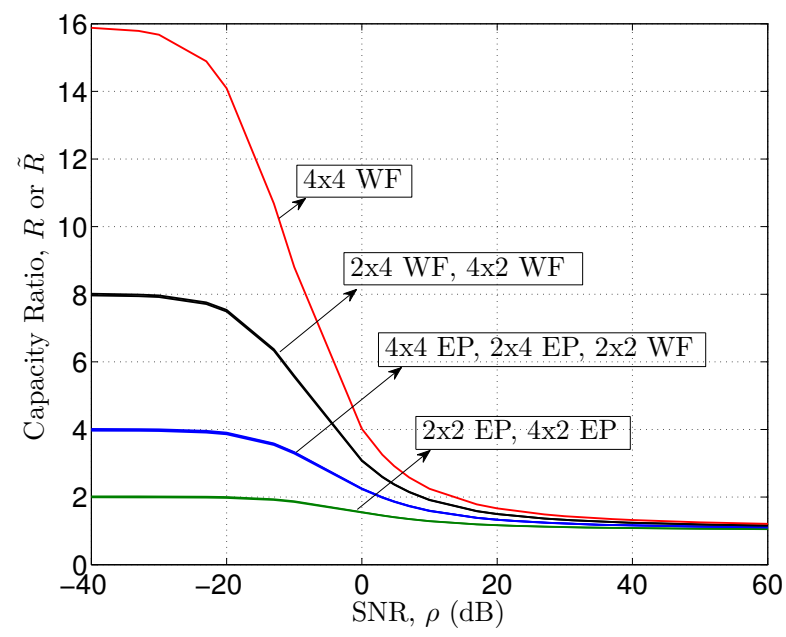

Fig. 5. $\quad R$ or $\tilde{R}$ for a fully correlated channel.

\section{CONCLUSION}

We have presented new results for capacity improvement in MIMO systems in the form of the MIMO-SISO capacity ratio

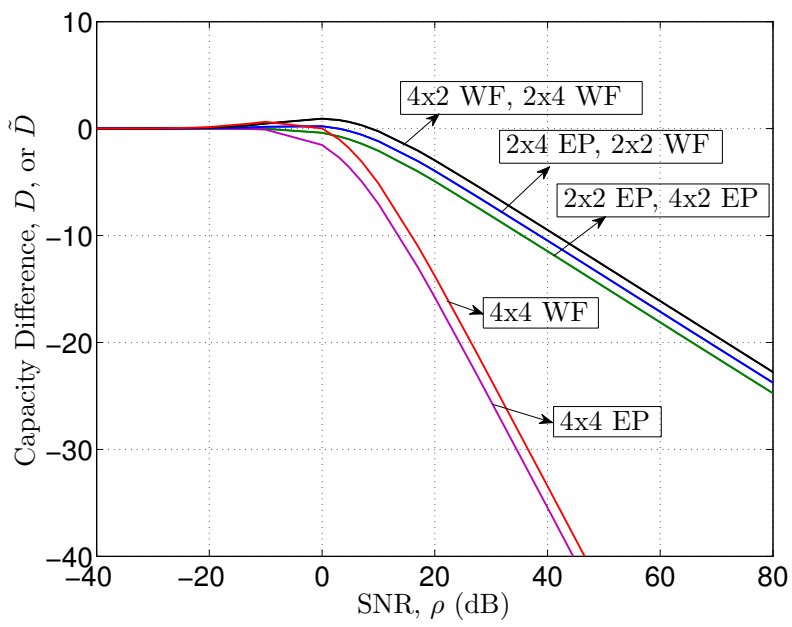

Fig. 6. $\quad D$ or $\tilde{D}$ for a fully correlated channel.

and difference. Limiting results for the two capacity metrics have been derived and their variation with SNR is shown. In particular, we observe the evolution from a power gain regime at low SNR to a degrees of freedom gain regime at high SNR. This evolution over the SNR range from $-5 \mathrm{~dB}$ to $20 \mathrm{~dB}$ is particularly important as it is commonly observed in cellular systems. In addition, we derive an asymptotic expression for the capacity difference at high SNR and show that it settles to a constant value (positive or negative) that depends upon antenna numbers and channel conditions.

\section{REFERENCES}

[1] E. Telatar, "Capacity of multi-antenna Gaussian channels," European Transactions on Telecommunications, vol. 10, no. 6, pp. 585-595, 1999.

[2] G. Foschini and M. Gans, "On limits of wireless communications in a fading environment when using multiple antennas," Wireless Personal Communications, vol. 6, no. 3, pp. 311-335, 1998.

[3] A. Goldsmith, S.A.Jafar, N. Jindal, and S. Vishwanath, "Capacity limits of MIMO channels," IEEE J. Sel. Area Comm., vol. 21, no. 5, pp. 684 702, 2003.

[4] J. Andersen, "Array gain and capacity for known random channels with multiple element arrays at both ends," IEEE J. Sel. Areas Comm., vol. 18, no. 11 , pp. $2172-2178,2000$.

[5] S. Loyka, "Channel capacity of MIMO architecture using the exponential correlation matrix," IEEE Commun. Let., vol. 5, no. 9, pp. 369-371, 2001.

[6] D. Shiu, G. Foschini, M. Gans, and J. Kahn, "Fading correlation and its effect on the capacity of multielement antenna systems," IEEE Trans. Commun., vol. 48, no. 3, pp. 502-513, 2000.

[7] P. Smith, S. Roy, and M. Shafi, "Capacity of MIMO systems with semicorrelated flat fading," IEEE Trans. Inf. Theory, vol. 49, no. 10, pp. 2781-2788, 2003.

[8] M. Chiani, M. Win, and A. Zanella, "On the capacity of spatially correlated MIMO Rayleigh-fading channels," IEEE Trans. Inf. Theory, vol. 49 , no. 10 , pp. 2363-2371, 2003.

[9] H. Shin and J. Lee, "Capacity of multiple-antenna fading channels: Spatial fading correlation, double scattering, and keyhole," IEEE Trans. Inf. Theory, vol. 49, no. 10, pp. 2636-2647, 2003.

[10] — "Closed-form formulas for ergodic capacity of MIMO Rayleigh fading channels," in IEEE International Conference on Communications, vol. 5, 2003, pp. 2996-3000.

[11] D. Tse and P. Viswanath, Fundamentals of Wireless Communication. Cambridge Univ Press, 2005.

[12] A. Zanella, M. Chiani, and M. Win, "On the marginal distribution of the eigenvalues of Wishart matrices," IEEE Trans. Commun., vol. 57, no. 4, pp. 1050-1060, 2009. 